\title{
Ronald J. Gillespie; the VSEPR model; and molecular symmetry
}

\author{
István Hargittai
}

Published online: 4 March 2009

(C) Springer Science+Business Media, LLC 2009

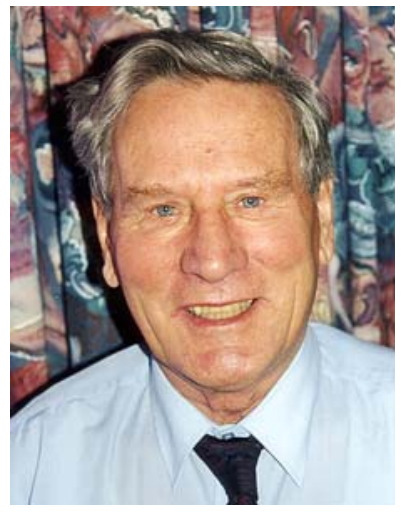

Fig. 1 Ronald J. Gillespie, 1998 (photograph by I. Hargittai)

Ronald J. Gillespie (Fig. 1) of superacid and VSEPR fame is 85 years old this year. The community of chemists-in particular in inorganic chemistry and structural chemistry-honors him in many ways. Ron-as he is known to many-has been both a great research chemist and a chemistry educator. His pioneering work in superacids has been recognized and might have even earned him a share of the Nobel Prize. Although it did not, nobody doubts that his contribution was of Nobel-prize-caliber. However, research achievements are almost always parts of a process; they build on previous achievements, on the one hand, and are superseded by others, on the other hand. Also, if one scientist does not make a certain discovery, another will,

\footnotetext{
I. Hargittai ( $\square)$

Department of Inorganic and Analytical Chemistry and Materials Structure and Modeling Research Group of the Hungarian, Academy of Sciences, Budapest University of Technology and Economics, P. O. Box 91, 1521 Budapest, Hungary e-mail: istvan.hargittai@gmail.com
}

sooner or later. Ron's great contribution with the Valence Shell Electron Pair Repulsion or VSEPR model (initially together with Ronald Nyholm, who soon died, much prematurely) is of a different character. The discovery of the VSEPR model was not inevitable and, although it relied on previous notions of trends in the variations in inorganic molecular structures, it was more unique and more personal than most scientific discoveries. The essence of the model is that the repulsion interactions of all electron pairs, counting both shared and unshared ones, in the valence shell of the central atom in a molecule determines the shape of the molecule. This model then was augmented with a few principal rules pertaining to the effects of multiple bonds, ligand electronegativity, and d-orbital availability, but its basic tenet has remained its cornerstone.

As a student, I had no knowledge of this model, and much of the information about the shapes of molecules we had to learn one by one. Once in possession of the model, many of the structural variations could be inscribed into the realm of a set of rudimentary rules. I was introduced to the model in the discussion following my first ever seminar presentation in the United States. It was in January 1969; I was on my way to the University of Texas at Austin, and was invited to Indiana University in Bloomington by Russel A. Bonham. I talked about the structures of some simple molecules containing sulfur as we determined them by gas-phase electron diffraction in Budapest. I was trying to observe trends in the structural variations and even interpret them, when Russ asked whether I had tried to apply VSEPR considerations to them. I was taken by this model so much that I penned a long letter to my wife-andgraduate student back in Budapest, and illustrated it with loops of electron pairs in color (the new knowledge of this attractive model kept me up all night following my seminar, or it may have been just jet leg). 
When, after 1 year, I returned home, I wrote a long article in a popular-science magazine about the model, but doing so, I ran into difficulties. As I tried to use the simplest molecules to illustrate the applicability of the model, I observed conspicuous discrepancies, and this prolonged the completion of the article. For example, the model in its original formulation predicted a gradual decrease of the bond angles in the series of molecules $\mathrm{CF}_{4} \rightarrow \mathrm{NF}_{3} \rightarrow \mathrm{OF}_{2}$ just as was observed in $\mathrm{CH}_{4} \rightarrow \mathrm{NH}_{3} \rightarrow \mathrm{OH}_{2}$. While there was a decrease in $\mathrm{CF}_{4} \rightarrow \mathrm{NF}_{3}$, this was not the case in $\mathrm{NF}_{3} \rightarrow \mathrm{OF}_{2}$. The deviation from prediction was similar in $\mathrm{SiF}_{4} \rightarrow \mathrm{PF}_{3} \rightarrow \mathrm{SF}_{2}$ and even more conspicuous in $\mathrm{CCl}_{4} \rightarrow \mathrm{NCl}_{3} \rightarrow \mathrm{OCl}_{2}$ and in $\mathrm{SiCl}_{4} \rightarrow \mathrm{PCl}_{3} \rightarrow \mathrm{SCl}_{2}$.

I wrote a letter to Gillespie about my observations, which he could not resolve either, but suggested not rushing into publishing anything about them, because he was just bringing out his book about his model, and advertising problems about it at this time would not be fortunate. Years of frustrations followed, culminating in some quantum chemical calculations, which pointed to the necessity of more complete considerations of the interactions among all electron pairs. This meant following the variations not only of the bond angles, but also the angles involving the lone pairs of electrons. This consideration did not necessitate the introduction of any additional rule; it only meant a fuller assessment of the very interactions the VSEPR model was based on in the first place. Needless to say, Ron was not enthusiastic about augmenting the considerations that went beyond the original formulation.

The inspection of the results of our quantum chemical calculations in terms of electron density distributions of electron pairs, whether lone pairs, bonding pairs to ligands of different electronegativities, or multiple bonds, led to an interesting observation. The differences appeared minute in comparison with the observed structural variations. Looking back, one might conclude that had these calculations preceded any ideas about establishing a model, the VSEPR model might have never been formulated. This is not to say that in hindsight, there have not been attempts putting the VSEPR model onto a solid quantum mechanical foundation. There have even been suggestions to rename it Pauli Mechanics to stress the relationship of repulsion interaction with the Pauli Exclusion Principle. However, the physical basis of the VSEPR model may be expressed in much more rudimentary terms (Fig. 2).

Consider the potential energy expression

$V_{i j}=k / r_{i j}^{n}$

where $k$ is a constant, $r_{i j}$ is the distance between the points $i$ and $j$, and the exponent $n$ is large for strong and small for weak repulsion interactions. These interactions are generally much stronger than simple electrostatic coulomb

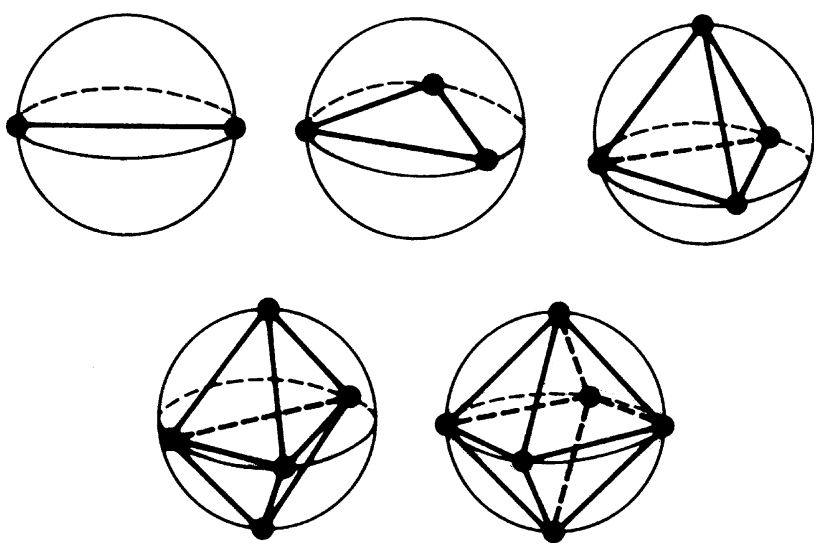

Fig. 2 The simplest shapes of arrangements of electron pairs in valence shells from a points-on-the-sphere model

interactions, but $n$ is not really known. This is though not an impediment to the broad application of the model, because when $n$ is larger than 3, the results become rather insensitive to the value of $n$. This insensitivity to the choice of $n$ is what provides the wide applicability of the VSEPR model (Fig. 3).

I stayed in mail-contact with Ron throughout these years, and we met in person for the first time only in the early 1990s. In the mid-1980s, I raised the question about a new book on the VSEPR model, and it turned out that my suggestion coincided with Ron's thinking about a new book as well. We decided to produce it jointly and the roles were clearly if tacitly defined from the start. I was to be the junior author doing much of the writing and Ron's role would be polishing what I wrote. This became a rewarding experience in that I learned that every single page must be didactically perfect-we were aiming at producing a textbook. It was also a frustrating experience as Ron was on sabbatical leaves most of the time; sailing the inter-coastal waterways on the East Coast of the United States and I had to be vigilant to make sure that my ever improving drafts would be delivered in the right marina at the right time.

Our joint writing was also made difficult by Ron's jealously guarding his model from any attempts of showing its limitations. One of those limitations was the model losing its validity with increasing ligand size relative to the size of the central atom. It was a little triumph when some regularity in non-bonded distances could be mentioned in the book. The irony of this is that years later, Ron became enamored with the importance of non-bonded interactions in determining molecular shapes.

Another, yet more general problem was that Ron was and has always been somewhat embarrassed by the tremendous success of his seemingly unsophisticated model and would have preferred to see its sophistication enhanced. So he wanted to stress the quantum mechanical 
Fig. 3 Collection of principal structure types predicted by VSEPR rules: bond configurations with two, three, four, five, and six electron pairs in the valence shell of the central atom
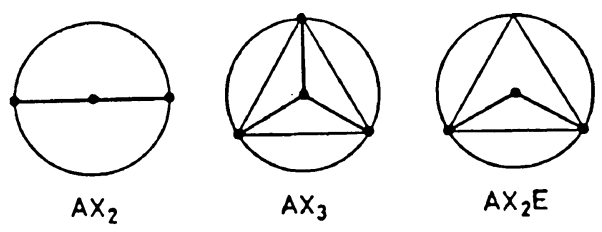

$A X_{2} E$<smiles>FB(F)[Sn]1(Cl)CCC1</smiles>

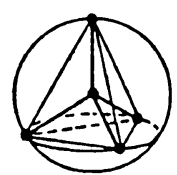

$\mathrm{AX}_{4}$

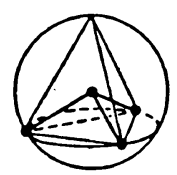

$A X_{3} E$

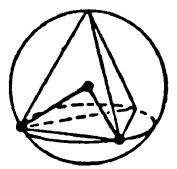

$A X_{2} E_{2}$<smiles>C</smiles><smiles>C1CCNC1</smiles><smiles>C1CCOC1</smiles>
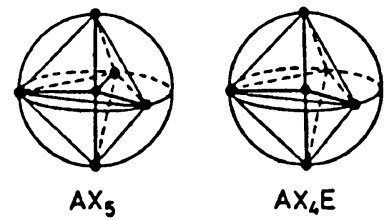<smiles>FP(F)(F)(F)CCS(F)(F)(F)(F)F</smiles>

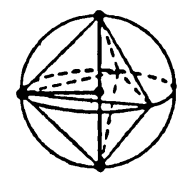

$A X_{3} E_{2}$
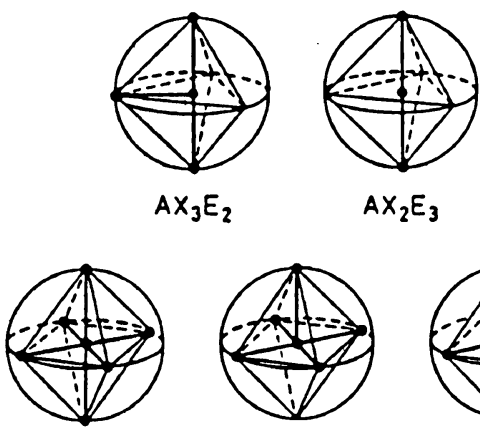

$A X_{6}$

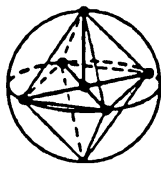

$\mathrm{AX}_{5} \mathrm{E}$

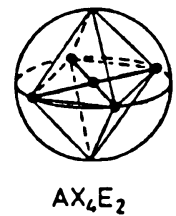

foundations of the model, which would have made it into a theory and, also, less applicable without involved computations. In contrast, if one would have to carry out quantum chemical computations each time instead of applying simple VSEPR considerations, the utility of the model would fast disappear. To me the highest compliment was when Ron told me that by the time we completed the book-it took almost 5 years for us-I may have known more about it than he. What I took away from the experience was what I learned about didactics from the great pedagogue that Ron has been and my pledge never get involved in co-authoring a book with anybody again (family members excepted).

The VSEPR model has had double significance. One is in chemistry education and the other is as a research tool. It is very difficult if at all possible to assess the importance of the VSEPR model in education. It has certainly made it easier for students at any level of chemistry instruction to grasp the notion of and variations in molecular structure, meaning the geometry of molecules in this case. Although the Nobel Prize should not be made into any kind of absolute measure, in a way it has become one, and had Gillespie's VSEPR model been awarded one, I doubt it would have elicited any protest. In fact, it could have been among the recently rather rare pure chemistry awards countering the growing tendency of using chemistry Nobel awards for achievements in discoveries of biological importance. The criteria for the Prize according to Alfred Nobel's Will have double requirements. One is a general stipulation referring to having conferred the greatest benefit on mankind. The other is specific to each of the five categories although the distinctions are slight. For chemistry it sounds, "to the person who shall have made the most important chemical discovery or improvement." The VSEPR model has benefited mankind in facilitating the dissemination of chemical literacy, and it was a discovery or at least an improvement of significance. Chemistry is not an easy subject; neither is it among the favorite ones for most pupils. Yet Gillespie's model certainly made its important aspects more user-friendly. If this is true to any extent, and I believe it is to a great extent, it has been beneficial to mankind.

The VSEPR model has been an educational tool more than a research tool, but its value as the latter should not be ignored. Only a couple of examples will be mentioned here in passing, but they are the more significant by the fact that 

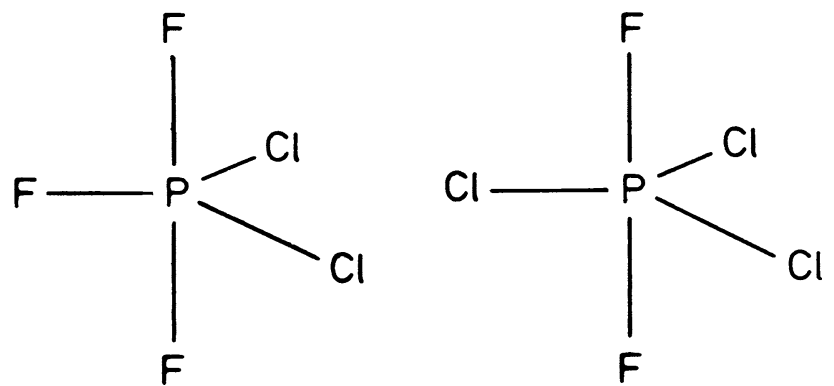

Fig. 4 Simple models of $\mathrm{OPF}_{3}\left(C_{3 v}\right.$ symmetry $)$ and $\mathrm{OClF}_{3}\left(C_{s}\right.$ symmetry)

Gillespie used his model in arguing against seemingly reliable computational work or seemingly incontrovertible experimental observations. When the structure of xenon hexafluoride was reported to have regular octahedral, $O_{h}$, symmetry from molecular orbital calculations, he suggested that it should be distorted rather than regular, due to a seventh electron pair in the xenon valence shell, which was a lone pair of electrons. A careful experimental study then provided direct evidence that the structure was indeed distorted rather than regular. When the structure of the then newly produced $\mathrm{OClF}_{3}$ molecule was reported to have $C_{3 v}$ symmetry, based on spectroscopic evidence, the same as the analogous $\mathrm{OPF}_{3}$, Gillespie argued that $\mathrm{OClF}_{3}$ should have lower symmetry $\left(C_{s}\right)$ than $\mathrm{OPF}_{3}$ due to the presence of an additional electron pair in the chlorine valence shell (Fig. 4). He virtually sent back the spectroscopists to their spectrometer. An extended spectrum then displayed additional transitions, hence lower symmetry, in full agreement with the VSEPR model.

Symmetry is an interesting aspect of molecular structure and above we have already seen that the relationship between molecular symmetry and VSEPR-based predictions is more complex than one might have assumed from the basic principles of the model. When there are two, three, four, and six equivalent electron pairs in the valence shell, the respective linear, symmetrical trigonal, regular tetrahedral, and regular octahedral arrangements are also the geometries of the highest possible symmetry, and there is no ambiguity about them. However, in case of five electron pairs, the trigonal bipyramidal arrangement is only slightly more advantageous than the tetrahedral pyramidal one, and for seven electron pairs the ambiguities are yet more enhanced. Intuitively, the trigonal bipyramidal $\mathrm{PF}_{2} \mathrm{Cl}_{3}$ and $\mathrm{PF}_{3} \mathrm{Cl}_{2}$ structures both should have the three equivalent bonds in the equatorial plane and the two-ones axially. Yet, again, considering VSEPR rules, in this case the difference between space requirements of electron pair domains for ligands of different electronegativities have to be taken into account. The axial positions are more crowded than the equatorial positions, hence bonds leading to
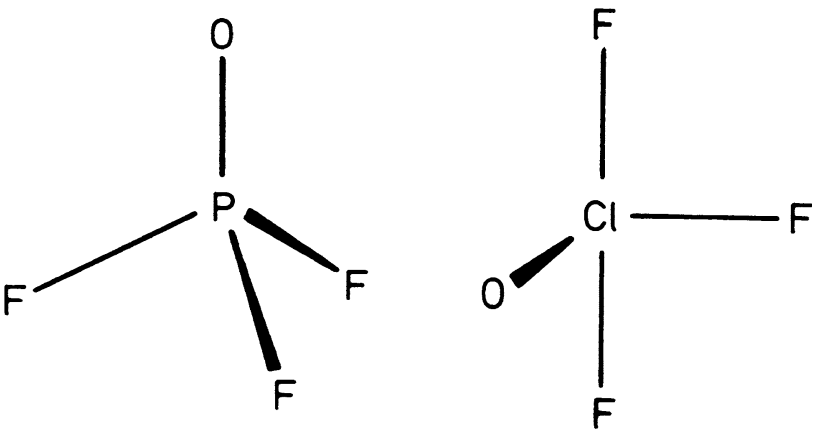

Fig. 5 Simple models of $\mathrm{PF}_{3} \mathrm{Cl}_{2}\left(C_{2 v}\right.$ symmetry $\left.)\right)$ and $\mathrm{PF}_{2} \mathrm{Cl}_{3}\left(D_{3 h}\right.$ symmetry)

more electronegative ligands will be axial and vice versa. Accordingly, the distribution of ligands among axial and equatorial positions will lead to $C_{2 v}$ symmetry for $\mathrm{PF}_{3} \mathrm{Cl}_{2}$ and $D_{3 h}$ for $\mathrm{PF}_{2} \mathrm{Cl}_{3}$ (Fig. 5).

Concerning molecular symmetry, it has no limits in principle, whereas, in classical crystallography, crystal symmetries excluded five-fold symmetry. Following the discovery of quasicrystals and in terms of generalized crystallography, there has been an expansion of recognized symmetry possibilities in condensed state structures as well—of course, they have always been present in nature. It is noteworthy, however, that different symmetries may occur with different frequencies and play different roles in structures. Thus, for example, symmetry planes may hinder maximum space utilization in the condensed phase, that is, when the symmetry plane would relate two molecules, resulting in considerable empty space between them. The presence of two-fold symmetry, on the other hand, facilitates space utilization. In molecular symmetries there is no such preference and the symmetry plane carries no disadvantage either, and has a high occurrence, especially in the structures of simple molecules. Molecular symmetries may have great importance for biological function, although this has yet been understood in few cases only. The most conspicuous example is the two-fold symmetry of the double helix structure of DNA directly related to the copying mechanism for this molecule. In contrast, the twofold symmetry of the molecule of the photosynthetic reaction center has been admired, but its function, if it has one at all, has not yet been understood. Symmetry is a most useful concept and it may have functional utility, but when observed in molecules, especially in small molecules, it may just be an expression of equalities of interactions between identical parts of the system. It may be counterproductive to read too much into it in most cases. Symmetry considerations and the structural consequences of VSEPR often point in the same direction and both are helpful in systematizing structures and understanding their variations. With the VSEPR model, Ronald Gillespie 
placed a most useful tool into the hands of chemistry educators and researchers and for this and his other contributions we celebrate him on the occasion of his remarkable anniversary. It is instructive to review his path to his achievements.

Ron was born in London in 1924. When he was 11 years old, he was awarded a scholarship to the local grammar school; this eased the burden of paying for his education, but his parents, who were not very well off, still had to pay for school uniforms, sports clothing, and train fares. He was an average student during the term, but his performance was outstanding on the final exams, especially in science. In preparation for university, he studied chemistry, physics, pure mathematics, and applied mathematics. He was much better in chemistry and physics than in mathematics, but he worked hard to bring up his math. His physics teacher gave boring lectures by simply reading the textbook aloud, but the chemistry teacher gave them a lot of exciting lab work. Ron graduated from school in 1942 and started a 2-year chemistry course at University College London. They were evacuated to the University of Wales in Aberystwyth at the beginning of Ron's studies. Toward the end of the second year, as they were moving back to London, the head of the chemistry department, Christopher Ingold asked him whether he would like to stay and do research. Thus, Gillespie became a member of Ingold's team working on aromatic nitration. Ron made remarkable progress in his research when he found cryoscopic evidence for the nitronium ion. The interaction with Ingold was infrequent; Ingold came by the lab once a month, in the company of Ted Hughes; he was taciturn; but even his remoteness appeared inspiring. Having worked with the nitronium ion, Gillespie worked a lot with sulfuric acid, leading his interest toward other superacids. Ingold let him working quite independently, but helped him writing up his papers. Several of them were actually written by Ingold, but appeared under Gillespie's name as sole author. Not long before Gillespie got his Ph.D. degree in 1949, Ingold casually asked him to give a course on molecular properties, which meant also an appointment as Assistant Lecturer at the University.

In 1953, Gillespie got a Commonwealth Fund Fellowship to work in the United States; he spent a year at Brown University; and toured the United States and Canada at the end of the year. It was then that the idea of moving to North America occurred to him. What Gillespie perceived before his American sojourn as encouragement, upon having returned to University College, he felt that Ingold's tight control of everything was hindering advancement. He was trying to strike out in new directions and was moving increasingly into inorganic chemistry in 1957. At the same time, he developed a fruitful interaction with Ronald $\mathrm{Ny}$ holm, a newly appointed faculty member from Australia. Their discussions led to a joint review of inorganic stereochemistry in which Gillespie dealt with the main groups and Nyholm with the transition metals. Working on this review inspired Gillespie to formulate the rules that later became the VSEPR model. On another trip to North America, he visited McMaster University, which was on its way of rapidly developing into a major research school from a small Baptist college. They made him an offer, which he could not refuse; his package contained double of his London salary, a Raman spectrometer, and an NMR spectrometer. In London, under Ingold, instrumentation was compartmentalized under different individuals, and there was not even a good NMR unit that Gillespie might have had access to.

Gillespie moved to Canada in 1958 and has stayed at McMaster University ever since. He was elected Fellow of the Royal Society of Canada in 1965 and Fellow of the Royal Society (London) in 1977. His research over the decades concentrated on superacids, sulfuric acid, fluorosulfuric acid, and hydrogen fluoride, and generally, fluorine chemistry. As for the VSEPR model, he was more of a promoter than doing extensive research in it. He also became engaged in textbook writing for general chemistry. In recent decades, the VSEPR model has taken off, and is often mentioned in the literature without even referring to Gillespie. This is the fate of important discoveries as the Periodic Table often figures without Mendeleev's name or the double helix without spelling out Watson and Crick. The expression VSEPR and Gillespie's name have become synonyms.

\section{Bibliography}

Gillespie RJ, Hargittai I (1991) The VSEPR model of molecular geometry. Allyn \& Bacon, Boston

Hargittai I (2002, 2003) The road to Stockholm: Nobel Prizes, science, and scientists. Oxford University Press, Oxford, UK

Hargittai I (2003) Candid science III: more conversations with famous chemists. Imperial College Press, London

Hargittai M, Hargittai I (2009) Symmetry through the eyes of a chemist, 3rd edn. Springer, London 